PROCEEDINGS OF THE

AMERICAN MATHEMATICAL SOCIETY

Volume 131, Number 6, Pages 1771-1776

S 0002-9939(02)06706-0

Article electronically published on October 1, 2002

\title{
MASS POINTS OF MEASURES ON THE UNIT CIRCLE AND REFLECTION COEFFICIENTS
}

\author{
LEONID GOLINSKII
}

(Communicated by Andreas Seeger)

\begin{abstract}
Measures on the unit circle and orthogonal polynomials are completely determined by their reflection coefficients through the Szegö recurrences. We find the conditions on the reflection coefficients which provide the lack of a mass point at $\zeta=1$. We show that the result is sharp in a sense.
\end{abstract}

\section{INTRODUCTION}

Let $\mathcal{P}$ be the set of all probability measures $\mu$ on the unit circle $\mathbb{T}=\{|\zeta|=1\}$ with infinite support. The latter is defined as the smallest closed set with the complement having $\mu$-measure zero. The polynomials $\phi_{n}(z)=\kappa_{n} z^{n}+\ldots$, orthonormal on the unit circle with respect to $\mu$ are uniquely determined by the requirement that $\kappa_{n}>0$ and

$$
\int_{\mathbb{T}} \phi_{n}(\zeta) \overline{\phi_{m}(\zeta)} d \mu=\delta_{n, m}, \quad n, m=0,1, \ldots, \zeta \in \mathbb{T} .
$$

The monic orthogonal polynomials $\Phi_{n}$ are $\Phi_{n}(z)=\kappa_{n}^{-1} \phi_{n}(z)=z^{n}+\ldots$, and the values $a_{n}=a_{n}(\mu) \stackrel{\text { def }}{=} \Phi_{n}(0)$ are known as the reflection coefficients.

Let us recall that the orthogonal polynomials (both monic and orthonormal) as well as the measure itself are completely determined by their reflection coefficients through the Szegö recurrences

$$
\Phi_{n}(z)=z \Phi_{n-1}(z)+a_{n} \Phi_{n-1}^{*}(z), \quad n \in \mathbb{N} \stackrel{\text { def }}{=}\{1,2, \ldots\}, \Phi_{0}=1
$$

(cf. [5, formula (11.4.7), p. 293]), and the connection between the reflection coefficients and the leading coefficients $\kappa_{n}$ is given by

$$
\kappa_{n}^{2}=\prod_{k=1}^{n}\left(1-\left|a_{n}\right|^{2}\right)^{-1}, \quad n \in \mathbb{N}, \kappa_{0}=1
$$

(cf. [1, formula (8.6), p. 156]). Here the reversed *-polynomial of a polynomial $p_{n}$ of degree $n$ is defined by $p_{n}^{*}(z) \stackrel{\text { def }}{=} z^{n} \overline{p_{n}(1 / \bar{z})}$. Moreover, each sequence $a_{n}$ of points from the open unit disk $\mathbb{D}$ comes up as a sequence of reflection coefficients for a

Received by the editors December 13, 2001 and, in revised form, January 14, 2002.

2000 Mathematics Subject Classification. Primary 42C05.

Key words and phrases. Measures on the unit circle, orthogonal polynomials, Szegő recurrence relations.

This material is based on work supported by the INTAS Grant 2000-272.

(C)2002 American Mathematical Society 
certain uniquely determined probability measure $\mu$. Hence, we have some sort of parametrization of the set $\mathcal{P}$ with free parameters from $\mathbb{D} \times \mathbb{D} \times \ldots$.

The problem we study in the present paper is the relation between the existence of a mass point at $\zeta=1$ and behavior of the reflection coefficients. The argument here relies upon the equivalence

$$
\mu\{\zeta\}>0, \quad \zeta \in \mathbb{T} \Longleftrightarrow \sum_{n=0}^{\infty}\left|\phi_{n}(\zeta)\right|^{2}<\infty
$$

(cf. 4, pp. 45-46], [2, § 20]). Note that within the Szegő class of measures, which can be defined by the condition $\sum_{n=0}^{\infty}\left|a_{n}\right|^{2}<\infty$, the sequence $\left\{\kappa_{n}\right\}$ is increasing and bounded, and hence

$$
\sum_{n=0}^{\infty}\left|\phi_{n}(\zeta)\right|^{2}<\infty \Longleftrightarrow \sum_{n=0}^{\infty}\left|\Phi_{n}(\zeta)\right|^{2}<\infty .
$$

The starting point for us is the following result due to P. Nevai [3, Theorem 2.5].

Theorem. If the reflection coefficients $a_{n}$ are real for all $n$ and they are nonnegative for all but finitely many values of $n$, then the corresponding measure $\mu$ has no mass point at 1 .

An example below shows that the first assumption of this theorem (which is actually the subject of our investigation) cannot be discarded.

Example 1. Put

$$
a_{1}=\frac{-1+i}{2}, \quad a_{2}=\frac{-1-i}{2}, \quad a_{k}=\frac{1}{k}, \quad k \geq 3,
$$

which gives rise to some measure $\nu$ in the Szegö class. We have by (1) $\Phi_{1}(z)=z+a_{1}$, $\Phi_{2}(z)=z^{2}+\left(a_{1}+a_{2} \bar{a}_{1}\right) z+a_{2}$, so that

$$
\Phi_{1}(1)=1+a_{1}=\frac{1+i}{2}, \quad \Phi_{2}(1)=1+a_{1}+a_{2}+a_{2} \bar{a}_{1}=\frac{i}{2},
$$

which is a pure imaginary number. Now keeping in mind that $\Phi_{m}^{*}(1)=\overline{\Phi_{m}(1)}$, write (1) at $z=1$ :

$$
\Phi_{n}(1)=\Phi_{n-1}(1)+a_{n} \overline{\Phi_{n-1}(1)}, \quad n \in \mathbb{N} .
$$

After separating the real and imaginary parts in (4) and iterating up, we come to

$$
\begin{aligned}
& \Re \Phi_{n}(1)=\Re \Phi_{2}(1) \prod_{k=3}^{n}\left(1+a_{k}\right)=0, \\
& \Im \Phi_{n}(1)=\Im \Phi_{2}(1) \prod_{k=3}^{n}\left(1-a_{k}\right)=\frac{1}{2} \prod_{k=3}^{n}\left(1-\frac{1}{k}\right)=\frac{1}{n}, \quad n \geq 3 .
\end{aligned}
$$

It follows that $\Phi_{n}(1)$ is square summable and hence $\nu\{1\}>0$.

On the other hand, the following modification of Nevai's theorem holds.

Theorem 1. Let $\Re a_{n} \geq 0$ for all $n \in \mathbb{N}$ and

$$
\sum_{n=1}^{\infty} \prod_{k=1}^{n}\left(1-\left|\Im a_{n}\right|\right)^{2}=\infty .
$$

Then the corresponding measure $\mu$ has no mass point at 1. 
We start out with the following simple lemma.

Lemma 1. If $\Re a_{n} \geq 0$ for all $n \in \mathbb{N}$, then $\left|\Im \Phi_{n}(1)\right| \leq \Re \Phi_{n}(1)$.

Proof. We proceed by induction. It is clear that

$$
\Re \Phi_{1}(1)=1+\Re a_{1} \geq 1>\left|a_{1}\right|=\left|\Im \Phi_{1}(1)\right| .
$$

Suppose that the statement is true for $k=1,2, \ldots, n-1$. Put

$$
a_{n}=\alpha_{n}+i \beta_{n}, \quad \Phi_{n}(1)=\Re \Phi_{n}(1)+i \Im \Phi_{n}(1)=u_{n}+i v_{n}
$$

and write (4) as a system of two linear recurrences

$$
\begin{aligned}
& u_{n}=u_{n-1}+\alpha_{n} u_{n-1}+\beta_{n} v_{n-1}, \\
& v_{n}=v_{n-1}+\beta_{n} u_{n-1}-\alpha_{n} v_{n-1} .
\end{aligned}
$$

We want to show that $u_{n} \pm v_{n} \geq 0$. We have

$$
\begin{aligned}
u_{n}+v_{n} & =u_{n-1}\left(1+\alpha_{n}+\beta_{n}\right)+v_{n-1}\left(1-\alpha_{n}+\beta_{n}\right) \\
& \geq u_{n-1}\left(1+\alpha_{n}+\beta_{n}\right)-\left|v_{n-1}\right|\left|1-\alpha_{n}+\beta_{n}\right| .
\end{aligned}
$$

But $\left|1-\alpha_{n}+\beta_{n}\right| \leq 1+\alpha_{n}+\beta_{n}$ since

$$
\begin{gathered}
1+\alpha_{n}+\beta_{n}+1-\alpha_{n}+\beta_{n}=2+2 \beta_{n} \geq 0, \\
1+\alpha_{n}+\beta_{n}-1+\alpha_{n}-\beta_{n}=2 \alpha_{n} \geq 0
\end{gathered}
$$

by the assumption of Lemma 1 . Hence, $u_{n}+v_{n} \geq 0$.

The same reasoning applied to orthogonal polynomials generated by the sequence $\left\{\bar{a}_{n}\right\}$ leads to the second inequality $u_{n}-v_{n} \geq 0$.

Proof of Theorem 1. It is well known that all zeros of $\Phi_{n}$ lie inside $\mathbb{D}$ [1, p. 9], so that $\Phi_{n}(1) \neq 0$. By Lemma 1 this implies $u_{n}=\Re \Phi_{n}(1)>0$ for all $n \in \mathbb{N}$.

For $a_{n}=\alpha_{n}+i \beta_{n}$ put $\omega_{n} \stackrel{\text { def }}{=} \prod_{k=1}^{n}\left(1+\alpha_{k}\right) \geq 1$ and divide the first equation in (6) by $\omega_{n}$ :

Next, by Lemma 1

$$
\frac{u_{n}}{\omega_{n}}=\frac{u_{n-1}}{\omega_{n-1}}+\frac{\beta_{n}}{1+\alpha_{n}} \frac{v_{n-1}}{\omega_{n-1}} .
$$

$$
\frac{u_{n}}{\omega_{n}} \geq \frac{u_{n-1}}{\omega_{n-1}}-\frac{\left|\beta_{n}\right|}{1+\alpha_{n}} \frac{\left|v_{n-1}\right|}{\omega_{n-1}} \geq\left(1-\left|\beta_{n}\right|\right) \frac{u_{n-1}}{\omega_{n-1}} .
$$

Finally,

$$
u_{m} \geq \frac{u_{m}}{\omega_{m}} \geq \frac{u_{n-1}}{\omega_{n-1}} \prod_{k=n}^{m}\left(1-\left|\beta_{k}\right|\right), \quad m>n .
$$

The latter inequality along with (5) yields

$$
\sum_{n=0}^{\infty}\left|\Phi_{n}(1)\right|^{2} \geq \sum_{n=0}^{\infty} u_{n}^{2}=\infty
$$

The result follows immediately from (2) and (3).

Note that (5) holds as long as $\sum_{n=1}^{\infty}\left|\Im a_{n}\right|<\infty$.

Corollary. If the reflection coefficients $a_{n}$ satisfy $\Re a_{n} \geq 0$ for all $n \in \mathbb{N}$ and they are nonnegative for all but finitely many values of $n$, then the corresponding measure $\mu$ has no mass point at 1 .

It turns out that the first assumption in Theorem 1 is sharp in a sense. 
Theorem 2. For every $\epsilon>0$ there is a measure $\mu$ in the Szegö class with the reflection coefficients $a_{n}$ such that $\Re a_{n} \geq-\epsilon$ for all $n \in \mathbb{N}, a_{n} \geq 0$ for all sufficiently large $n$ and $\mu\{1\}>0$.

Proof. We follow the line of reasoning from the example above, but in a more sophisticated way. For $a_{n}=\alpha_{n}+i \beta_{n}$ put

$$
\alpha_{k}=-\alpha, \quad \beta_{k}=(-1)^{k} \alpha_{k}, \quad k=1,2, \ldots, 2 p ; \quad a_{k}=\frac{1}{k}, \quad k \geq 2 p+1 .
$$

We will show that the parameters $\alpha$ and $p$ can be found from the conditions

$$
0<\alpha<\epsilon, \quad u_{2 p}=\Re \Phi_{2 p}(1)=0 .
$$

To that end let us go back to (4) and write (6) in the matrix form (the matrix product is taken from right to left)

$$
\left[\begin{array}{l}
u_{n} \\
v_{n}
\end{array}\right]=\left(I+V_{n}\right)\left[\begin{array}{l}
u_{n-1} \\
v_{n-1}
\end{array}\right], \quad\left[\begin{array}{l}
u_{n} \\
v_{n}
\end{array}\right]=\prod_{1 \leq k \leq n}^{\curvearrowleft}\left(I+V_{k}\right)\left[\begin{array}{l}
1 \\
0
\end{array}\right],
$$

where $I$ is the $2 \times 2$ identity matrix and

$$
V_{k}=\left(\begin{array}{rr}
\alpha_{k} & \beta_{k} \\
\beta_{k} & -\alpha_{k}
\end{array}\right), \quad k=1,2, \ldots, n
$$

To meet $u_{2 p}=0$ we need to choose $p$ and $\alpha=\alpha_{p}$ in such a way that

$$
\prod_{1 \leq k \leq 2 p}^{\curvearrowleft}\left(I+V_{k}\right)=\left(\begin{array}{cc}
0 & * \\
* & *
\end{array}\right) .
$$

In our case for $1 \leq m \leq p$

$$
V_{2 m}=V_{+}=\left(\begin{array}{rr}
-\alpha & -\alpha \\
-\alpha & \alpha
\end{array}\right), \quad V_{2 m-1}=V_{-}=\left(\begin{array}{rr}
-\alpha & \alpha \\
\alpha & \alpha
\end{array}\right)
$$

and

Note also that

$$
\prod_{1 \leq k \leq 2 p}^{\curvearrowleft}\left(I+V_{k}\right)=\left[\left(I+V_{+}\right)\left(I+V_{-}\right)\right]^{p} .
$$

$$
V_{-}=J V_{+} J, \quad I+V_{-}=J\left(I+V_{+}\right) J, \quad J=\left(\begin{array}{rr}
-1 & 0 \\
0 & 1
\end{array}\right)
$$

so that our matrix product is just the power of a single matrix

$$
\prod_{1 \leq k \leq 2 p}^{\curvearrowleft}\left(I+V_{k}\right)=\left[\left(I+V_{+}\right) J\right]^{2 p}=U_{\alpha}^{2 p}, \quad U_{\alpha} \stackrel{\text { def }}{=}\left(\begin{array}{cc}
-1+\alpha & -\alpha \\
\alpha & 1+\alpha
\end{array}\right) .
$$

It is a matter of undergraduate linear algebra to find the eigenvalues of $U_{\alpha}$,

$$
\lambda_{1}=\alpha+\sqrt{1-\alpha^{2}}>1, \quad \lambda_{2}=\alpha-\sqrt{1-\alpha^{2}}<0
$$

(we assume that $\alpha<1 / 2$ and, hence, $\operatorname{det} U_{\alpha}=2 \alpha^{2}-1<0$ ), and to reduce $U_{\alpha}$ to diagonal form by means of a nonsingular transformation

$$
T=\left(\begin{array}{cc}
1 & 1 \\
y_{1} & y_{2}
\end{array}\right), \quad T^{-1}=\frac{1}{y_{2}-y_{1}}\left(\begin{array}{rr}
y_{2} & -1 \\
-y_{1} & 1
\end{array}\right)
$$


with

$$
\begin{aligned}
& y_{1}=\frac{1-\lambda_{2}}{1-\lambda_{1}}=\frac{1-\alpha+\sqrt{1-\alpha^{2}}}{1-\alpha-\sqrt{1-\alpha^{2}}}, \\
& y_{2}=\frac{1-\lambda_{1}}{1-\lambda_{2}}=\frac{1-\alpha-\sqrt{1-\alpha^{2}}}{1-\alpha+\sqrt{1-\alpha^{2}}}=y_{1}^{-1} .
\end{aligned}
$$

Finally, we arrive at the following expression for the object we are interested in:

$$
U_{\alpha}^{2 p}=T\left(\begin{array}{cc}
\lambda_{1}^{2 p} & 0 \\
0 & \lambda_{2}^{2 p}
\end{array}\right) T^{-1}=\frac{1}{y_{2}-y_{1}}\left(\begin{array}{cc}
\lambda_{1}^{2 p} y_{2}-\lambda_{2}^{2 p} y_{1} & * \\
* & *
\end{array}\right) .
$$

It remains only to choose $p$ and $\alpha$ to satisfy $\lambda_{1}^{2 p} y_{2}=\lambda_{2}^{2 p} y_{1}$ or, equivalently

$$
\left(\frac{\lambda_{2}}{\lambda_{1}}\right)^{2 p}=\frac{y_{2}}{y_{1}}, \quad\left(\frac{\sqrt{1-\alpha^{2}}-\alpha}{\sqrt{1-\alpha^{2}}+\alpha}\right)^{p}=\frac{\sqrt{1-\alpha^{2}}-1+\alpha}{\sqrt{1-\alpha^{2}}+1-\alpha}
$$

(note that both $y_{1}$ and $y_{2}$ are negative numbers).

Consider a transcendental equation

$$
\left(\frac{1-\tan x}{1+\tan x}\right)^{p}=\tan \frac{x}{2},
$$

which has a unique solution $x=x_{p}$ on the interval $(0, \pi / 4)$. It is clear that $x_{p} \rightarrow 0$ as $p \rightarrow \infty$. Put $\alpha_{p}=\sin x_{p}$ and pick $p$ to have $0<\alpha_{p}<\epsilon$. A routine calculation shows that

$$
\begin{aligned}
& \left(\frac{\sqrt{1-\alpha_{p}^{2}}-\alpha_{p}}{\sqrt{1-\alpha_{p}^{2}}+\alpha_{p}}\right)^{p}=\left(\frac{1-\tan x_{p}}{1+\tan x_{p}}\right)^{p}, \\
& \frac{\sqrt{1-\alpha_{p}^{2}}-1+\alpha_{p}}{\sqrt{1-\alpha_{p}^{2}}+1-\alpha_{p}}=\frac{\cos x_{p}-1+\sin x_{p}}{\cos x_{p}+1-\sin x_{p}}=\tan \frac{x_{p}}{2},
\end{aligned}
$$

so that both (8) and (7) hold.

Once this is done, the rest is clear in view of Example 1. Indeed, $\Phi_{2 p}(1) \neq 0$ now implies $\Im \Phi_{2 p}(1) \neq 0$, and for $n \geq 2 p+1$ we have

$$
\begin{aligned}
& \Re \Phi_{n}(1)=\Re \Phi_{2 p}(1) \prod_{k=2 p+1}^{n}\left(1+a_{k}\right)=0, \\
& \Im \Phi_{n}(1)=\Im \Phi_{2 p}(1) \prod_{k=2 p+1}^{n}\left(1-\frac{1}{k}\right)=\frac{2 p}{n} \Im \Phi_{2 p}(1),
\end{aligned}
$$

so that $\left\{\Phi_{n}(1)\right\}$ is a square summable sequence and $\mu\{1\}>0$.

Remark. Theorem 2 shows that for every $\epsilon>0$ there is a measure $\mu$ in the Szegö class with the reflection coefficients $a_{n}$ such that $\left|\Im a_{n}\right| \leq \epsilon$ for all $n \in \mathbb{N}, a_{n} \geq 0$ for sufficiently large $n$ and $\mu\{1\}>0$. Therefore, the first assumption in Nevai's theorem is also sharp in the same sense. 


\section{REFERENCES}

1. L. Ya. (aka Ya. L.) Geronimus, Orthogonal Polynomials: Estimates, asymptotic formulas, and series of polynomials orthogonal on the unit circle and on an interval, Consultants Bureau, New York, 1961. MR 24:A3469

2. Ya. L. Geronimus, Polynomials orthogonal on a circle and their applications, Series and Approximations, Amer. Math. Soc. Transl. (1), vol. 3, Providence, Rhode Island, 1962, pp. 178.

3. P. Nevai, Orthogonal polynomials, measures and recurrences on the unit circle, Trans. Amer. Math. Soc. 300 (1987), 175-189. MR 88d:42041

4. J. A. Shohat and J. D. Tamarkin, The Problem of Moments, Math. Surveys, vol. 1, Amer. Math. Soc., Providence, Rhode Island, 1970. MR 5:5c

5. G. Szegő, Orthogonal Polynomials, Fourth edition, Amer. Math. Soc. Colloq. Publ., vol. 23, Amer. Math. Soc., Providence, Rhode Island, 1975. MR 51:8724

Mathematics Division, Institute for Low Temperature Physics and Engineering, 47 Lenin Avenue, Kharkov 61103, Ukraine

E-mail address: golinskii@ilt.kharkov.ua 